\title{
IMPROVED ENERGY RATIO METHOD TO ESTIMATE COUPLING LOSS FACTORS FOR SERIES COUPLED STRUCTURE
}

\author{
Jintao $\mathrm{Gu}^{1}$ and Meiping Sheng ${ }^{2 *}$ \\ Northwestern Polytechnical University, Xi’an, China, 710072 \\ *Corresponding e-mail: smp@nwpu.edu.cn
}

\begin{abstract}
Energy ratio method (ERM), which is a widely used method for coupling loss factors (CLFs) measurement, may lead to numerical problems because of the inversion of a large dimension matrix. The improved energy ratio method proposed here simplified a series several-coupled system into a two-coupled system so that it becomes a two dimensions matrix instead of large dimensions one. Coupling loss factors of a series three-coupled structure are measured using this method. Vibrating responses of the structure has been tested and predicted with loss and coupling loss factors measured here in order to verify the accuracy of measured CLFs. The agreement of vibrating responses indicates the fact that the improved energy ratio method is of great accuracy and reliability.
\end{abstract}

Keywords: energy ratio method, coupling loss factors, series coupled system

\section{INTRODUCTION}

Statistical Energy Analysis (SEA), which has become quite widely used for studying the vibro-acoustic behavior of structures, vehicles, ships and buildings, is a popular and powerful technique for vibration analysis of complex structures at high frequencies ${ }^{1-4}$. In SEA, the structure is divided into subsystems and the response is described in terms of the total time average subsystem energies $E_{i}$ and input powers $P_{i}$. Coupling loss factors(CLFs) employed in SEA are defined to estimate the magnitude of transferred power. The power transfer terms have the form $\omega \eta_{i j} E_{i}$, where $E_{i}$ is the steady-state energy of subsystem $i, \omega$ is the central circle frequency of vibration, and $\eta_{i j}$ is the coupling loss factor from subsystem $i$ to subsystem $j$. CLFs describe three coupling relationship which are

(i) coupling between acoustic space and acoustic space,

(ii) coupling between acoustic space and structure,

(iii) coupling between structure and structure.

CLFs directly affect SEA prediction accuracy according to the power balance equation. If there exists some errors for CLFs estimation, the errors will be enlarged by the matrix inversion for the response prediction. Theoretical value of CLFs can be obtained in simple cases, but it is difficult for the real industrial structures. Thus, experimental investigation is the universal way to estimate CLFs.

Jianfei Yin $^{5}$ makes a great use of finite element methods and experimental statistical energy analysis to determine coupling loss factors for L-junctions comprised of homogenous and periodic ribbed plates. Thite $^{6}$ discussed two approaches for robust estimation of CLFs based on finite element method. Lyon ${ }^{7}$ gave estimates of the variance of CLFs and of the response of a subsystem, based on mode shape functions. Craik ${ }^{8}$ developed an alternative approach from observing that the distribution of the effective CLFs for individual realizations relative to the ensemble average was similar to the distribution of the point mobility of the receiver subsystem relative to its characteristic mobility. Maxit $^{9}$ calculated CLFs with a duel formulation and finite element model. He determined CLFs from the knowledge of the modes of the uncoupled subsystems and the modal damping. Manik $^{10}$ proposed a method of determining coupling loss factors by the power injection which is not affected by the strength of the coupling between subsystems, meanwhile Bies ${ }^{11}$ used inversion of the linear power balance equation to determine the plate loss factors and the coupling loss factors in situ. Nunes ${ }^{12}$ applied a fuzzy-set-based method to estimate CLFs. He paid attention to the influence of uncertain parameters in the finite element model used to estimate coupling loss factors. Cristina ${ }^{13}$ proposed a numerical method to obtain coupling loss factors in which the energies of an SEA system with two subsystems are obtained from deterministic numerical simulations. Ming $^{14}$ estimated coupling loss factors using structural intensity.

There are several other methods of investigating CLFs, SEA-like approach ${ }^{15}$, receptance method ${ }^{16}$, and point mobility method ${ }^{17}$.

Methods mentioned above have the following drawbacks:

(i) Some formulations or methods can not be widely used,

(ii) Some methods can only solve the problems of simple and typical structures,

(iii)Input power which is hard to obtain is needed for some methods.

Cuschieri ${ }^{18}$ proposed an experimental method namely energy ratio method (ERM) to get CLFs 
without input power, but his method may cause a numerical problem because there may be some illness in the matrix inversion involved in the computation. CLFs obtained by ERM can be negative which is inconsistent.

The approach presented in this paper is an improved energy ratio method based on a simplification of a series coupled structure into a two-subsystem coupled structure.

\section{COUPLING LOSS FACTORS FOR TWO-SUBSYSTEM COUPLED STRUCTURE}

Structure consists of two subsystems is shown in Fig. 1. The energy balance equation is given by

$$
\left(\begin{array}{cc}
p_{1} & 0 \\
0 & p_{2}
\end{array}\right)=\omega\left(\begin{array}{cc}
\eta_{11}+\eta_{12} & -\eta_{21} \\
-\eta_{12} & \eta_{21}+\eta_{22}
\end{array}\right)\left(\begin{array}{cc}
E_{1}^{(1)} & E_{1}^{(2)} \\
E_{2}^{(1)} & E_{2}^{(2)}
\end{array}\right)
$$

where $p_{i}(i=1,2)$ stands for input power injected into subsystem $i . E_{i}^{(j)}(i=1,2 . j=1,2)$ is vibration energy for subsystem $\mathrm{i}$ while subsystem $\mathrm{j}$ is excited. $\omega$ is circular frequency. $\eta_{i i}(i=1,2)$ is loss factor of subsystem $i$ and $\eta_{i j}(i=1,2 . j=1,2)$ is the coupling loss factor representing power flow from system $i$ to system $j$.

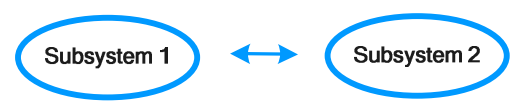

Figure 1. Structure consists of two subsystems

It can be readily shown that

$$
\left\{\begin{array}{l}
-\eta_{12} E_{1}^{(1)}+\eta_{21} E_{1}^{(1)}+\eta_{22} E_{2}^{(1)}=0 \\
\eta_{11} E_{1}^{(2)}+\eta_{12} E_{1}^{(2)}-\eta_{21} E_{2}^{(2)}=0
\end{array}\right.
$$

CLFs can be calculated with experimental results $\eta_{11}$,

$$
\begin{aligned}
& \eta_{22}, E_{1}^{(1)}, E_{2}^{(1)}, E_{1}^{(2)} \text { and } E_{2}^{(2)} \\
& \left\{\begin{array}{l}
\eta_{12}=\frac{\eta_{11} E_{1}^{(2)} E_{2}^{(1)}+\eta_{22} E_{2}^{(1)} E_{2}^{(2)}}{E_{1}^{(1)} E_{2}^{(2)}-E_{1}^{(2)} E_{2}^{(1)}}=\frac{\eta_{11} e_{1}+\eta_{22} e_{1} e_{2}}{e_{2}-e_{1}} \\
\eta_{21}=\frac{\eta_{22} E_{2}^{(1)} E_{1}^{(2)}+\eta_{11} E_{1}^{(2)} E_{1}^{(1)}}{E_{1}^{(1)} E_{2}^{(2)}-E_{1}^{(2)} E_{2}^{(1)}}=\frac{\eta_{22} e_{1}+\eta_{11}}{e_{2}-e_{1}}
\end{array}\right.
\end{aligned}
$$

where $e_{i}=\frac{E_{2}^{(i)}}{E_{1}^{(i)}},(i=1,2)$ are vibration energy ratio between subsystem 2 and subsystem 1 while only subsystem $i$ is excited.

\section{COUPLING LOSS FACTORS FOR SERIES COUPLED STRUCTURE}

SEA energy balance equation of structure consists of $n$ subsystems can be written as Eq. (4).

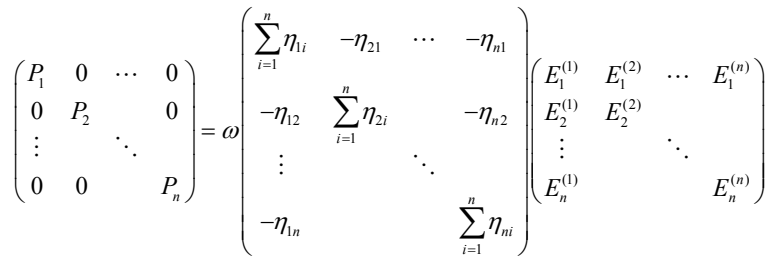

When subsystem $i$ is excited only, energy balance equation is given by

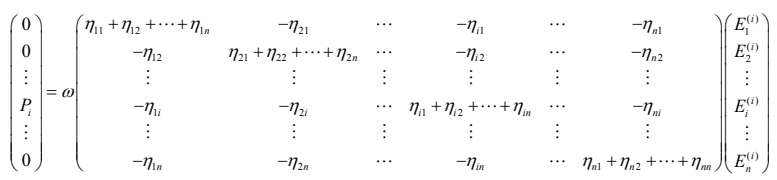

According formulation (5), it can be shown that

$$
\frac{P_{i}}{\omega}=\eta_{11} E_{1}^{(i)}+\eta_{22} E_{2}^{(i)}+\cdots+\eta_{n n} E_{n}^{(i)}
$$

In SEA applications, since indirect coupling loss factors can usually be ignored compared with direct coupling loss factors ${ }^{18}$, only direct coupling factors are necessary for computing structural vibration responses.

Fig. 2 shows the Series Coupled Structure built up by $n$ subsystems. Coupling loss factors between subsystem $i$ to subsystem $i+1 \quad(1 \leq i \leq n-1)$ will be calculated to introduce the method presented in this paper.

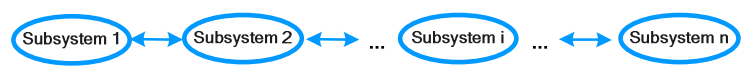

Figure 2. Series coupled structure

Rearranging Eq. (6),

$$
\begin{aligned}
\frac{P_{i}}{\omega} & =\eta_{11} E_{1}^{(i)}+\eta_{22} E_{2}^{(i)}+\cdots \eta_{i i} E_{i}^{(i)}+\eta_{i i+1} E_{i i+1}^{(i)}+\cdots+\eta_{n n} E_{n}^{(i)} \\
& =\eta_{i i}\left(\frac{\eta_{11}}{\eta_{i i}} E_{1}^{(i)}+\frac{\eta_{22}}{\eta_{i i}} E_{2}^{(i)}+\cdots+E_{i}^{(i)}\right)+\eta_{i i+1}\left(E_{i i+1}^{(i)}+\cdots+\frac{\eta_{n n}}{\eta_{i i+1}} E_{n}^{(i)}\right)
\end{aligned}
$$

Assume a new subsystem 1 which consists of subsystem $i$ and subsystems related to subsystem $i$ except subsystem $i+1$. The new subsystem 1 is built up with original subsystem $1 \sim$ subsystem $i$ in the series coupled structures. It is the same for new subsystem 2 which consists of subsystem $i+1 \sim$ subsystem $n$. Structure coupled by $n$ subsystems then becomes a two-subsystem coupled structure. Coupling loss factors between subsystem 1 and subsystem 2 can stand for coupling loss factors between subsystem $i$ and subsystem $i+1$. Loss factors of new subsystem 1 is the same as loss factors of subsystem $i$ while loss factor of 
new subsystem 2 can be represented by which of subsystem $i+1$, as expressed in formulation (7). The equivalent vibration energy of new subsystem 1 is

$$
\frac{\eta_{11}}{\eta_{i i}} E_{1}^{(i)}+\frac{\eta_{22}}{\eta_{i i}} E_{2}^{(i)}+\cdots+E_{i}^{(i)}
$$

The equivalent vibra tion energy can be understood as considering effects of subsystems which are connected directly and indirectly to subsystem $i$. The equivalent vibration energy of new subsystem 2 is $E_{i i+1}^{(i)}+\cdots+\frac{\eta_{n n}}{\eta_{i i+1}} E_{n}^{(i)}$, similarly.

Simplified system consists of the new subsystem 1 and subsystem 2 is shown in Fig. 3.

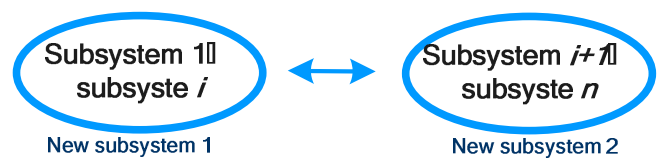

Figure 3. Simplified structure

Thus, $n$-coupled series structure becomes a two-coupled structure. Substitute loss factors and equivalent vibration energy of new subsystem 1 and 2 to Eq. (3), CLFs between new subsystem 1 and 2 can be calculated. The result stands for coupling loss factors between subsystem $i$ and subsystem $i+1$ of original system.

\section{EXPERIMENTAL INVESTIGATION}

The experiment is carried out on a shell connected by three shells, as shown in Fig. 4. Loss factors and vibration energies are tested in the process of measuring coupling loss factors. At first, loss factor of each subsystem will be estimated. Then, investigate vibration response for calculating vibration energy when every subsystem is excited alone. Experimental coupling loss factors of present method and ERM between adjacent subsystems are shown in Fig. 5 .

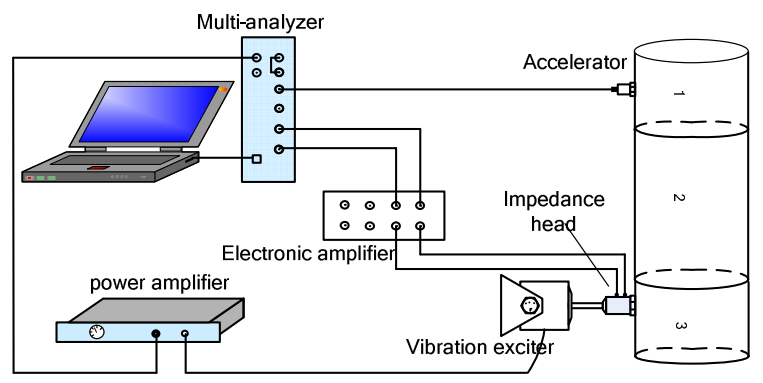

Figure 4. Experimental setup.
It is shown that there is no negative coupling loss factor calculated based on experimental data using improved method while $\eta_{21}$ and $\eta_{32}$ obtained by ERM are negative in some frequency bands.
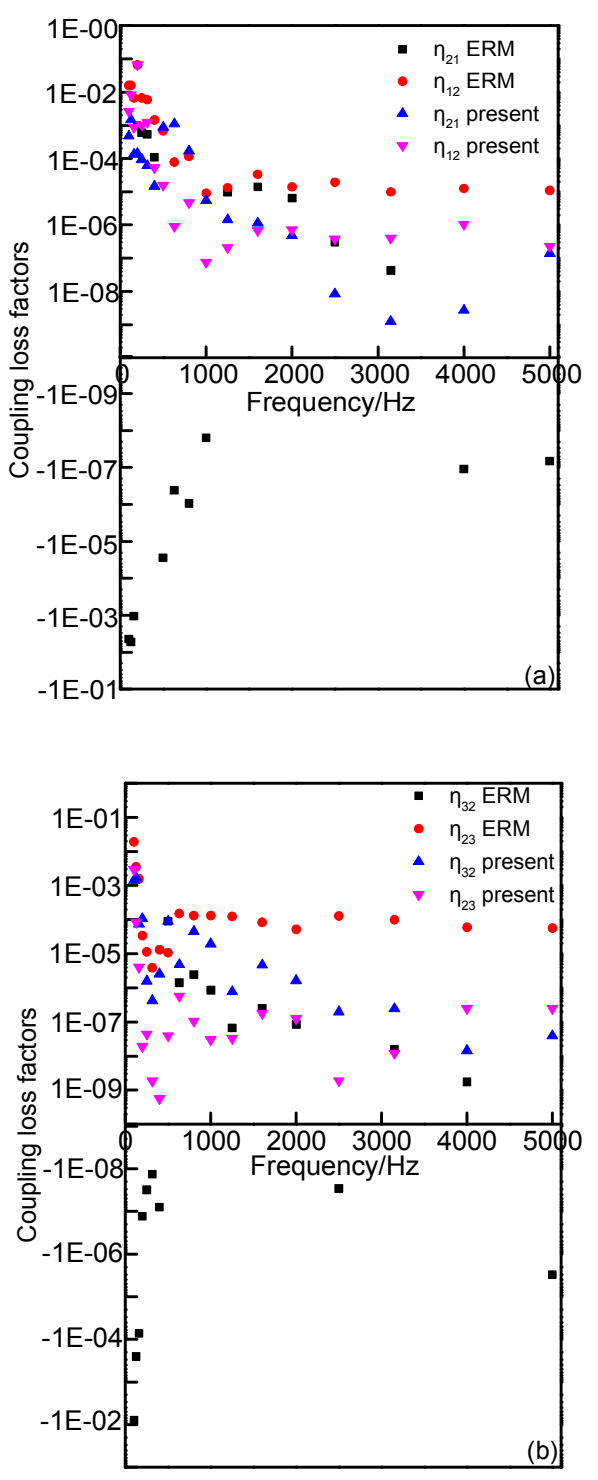

Figure 5. CLFs by proposed method: (a) $\eta_{21}$ and

$$
\eta_{12}, \text { (b) } \eta_{32} \text { and } \eta_{23} \text {. }
$$

An experimental verification with subsystem 3 excited only, has been carried out. To accomplish the experimental verification, a comparison of vibration energies of subsystem 1 and 2 between predicted and tested is needed. Predicted results can be obtained with vibration energy of subsystem 3, loss factors and coupling loss factors tested on section 4. Energies of subsystem 1 and 2 are shown in Fig. 6.

The good agreement of predicted and tested energies of subsystem 1 and 2 indicates that the improved method is of great accuracy and reliability. 

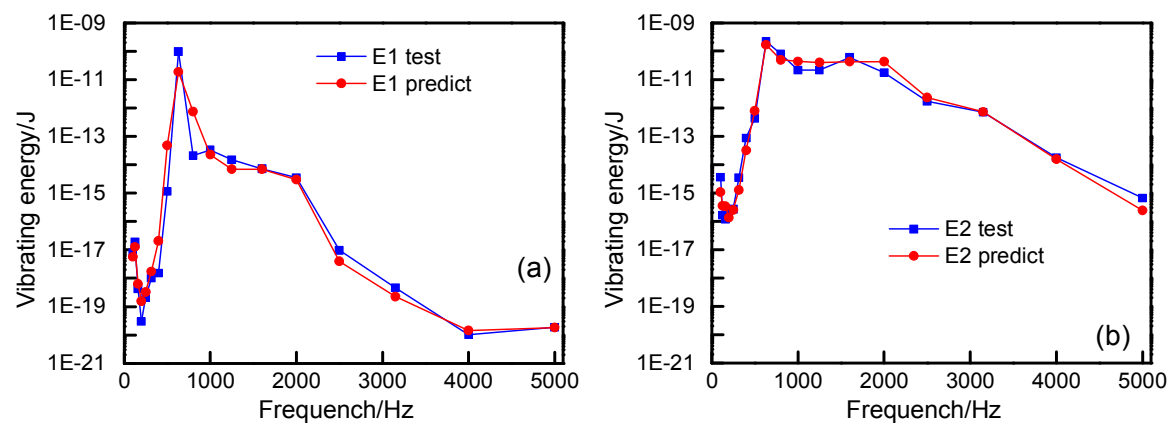

Figure 6. Vibrating energy of subsystem 1 and 2.

\section{CONCLUSION}

The improved energy ratio method, which simplifies series coupled structure into two-subsystem coupled structure, is proposed. CLFs for the simplified structure are all positive and reliable according to the experimental result. The comparison between experimental and predicted vibrating response which is calculated with coupling loss factors by improved energy ratio method. It is clear that the improved method can obtain positive coupling loss factors with satisfied accuracy and of great importance for SEA engineering application.

\section{REFERENCES}

1. Heckl M., and Lewit M., 1994, "Statistical energy analysis as a tool for quantifying sound and vibration transmission paths," Philosophical Transactions of the Royal Society of London Series A, 346, pp. 429-554.

2. Borello G., 1998,"Prediction and control of structure borne noise transfers in vehicles using SEA," Proceedings of Euro-Noise, 98, pp.183-188.

3. Hynna P., Klinge P., and Vuoksinen J., 1995, "Prediction of structure-borne sound transmission in large welded ship structures using statistical energy analysis," Journal of Sound and Vibration, 180, pp. 583-607.

4. Gibbs B.M., and Gilford C.L.S., 1976, "The use of power flow methods for the assessment of sound transmission in building structures," Journal of Sound and Vibration, 49, pp. 267-286.

5. Yin J. F., 2010, "Deterination of Coupling Loss Factors Between L-Junctions of Coupled Homogenous and Periodic Plates Using Finite Element Methods," 17 th International Congress on Sound and Vibration, ICSV, 4, pp. 2589-2596

6. Thite A.N., and Mace B.R., 2007, “ Robust estimation of coupling loss factors from finite element analysis," Journal of Sound and Vibration, 303, pp. 814-831

7. Lyon R.H., and DeJong R.G., 1995, "Theory and Application of Statistical Energy Analysis," 2nd Edition, Butterworth Heinemann, Boston.

8. Craik R.J.M., Steel J.A., and Evans D.I., 1991, "Statistical energy analysis of structure-borne sound transmission at low frequencies," Journal of Sound and
Vibration 144, pp. 95-107.

9. Maxit L., Guyader J.-L., 2001, "Estimation of SEA coupling loss factors using a dual formulation and FEM modal information, part I: theory," Journal of Sound and vibration, 239, pp. 907-930.

10. Manik D.N., 1998, "A New method for determining coupling loss factors for SEA," Journal of Sound and Vibration, 211, pp. 521-526.

11. Bies D.A., and Hamid S., 1980, "In situ determination of loss and coupling loss factors by the power injection method," Journal of Sound and Vibration, 70, pp. 187-204.

12. Nunes R.F., Ahmida K.M., and J.R.F. Arruda, 2007, “ Applying a fuzzy-set-based method for robust estimation of coupling loss factors," Journal of Sound and Vibration, 307 pp. 38-51.

13. Cristina D. C., Jordi P. P., and Antonio R F., 2013, "Numerical estimation of coupling loss factors in building acoustics," Journal of Sound and Vibration, 332, pp. 5433-5450

14. Ming R. S., 1998, “ The measurement of coupling loss factors using the structural intensity technique," Journal of the Acoustical Society of America, 103, pp. 401-407.

15. Mace B., 2005, "Statistical energy analysis: coupling loss factors, indirect coupling and system modes," Journal of Sound and Vibration, 279 pp. 141-170.

16. Shankar K., and Keane A.J., 1997, "Vibrational energy flow analysis using a substructure approach: the application of receptance theory to FEA and SEA," Journal of Sound and Vibration, 201, pp.491-513.

17. Cacciolati C., and Guyader J.L., 1994, "Measurement of SEA Coupling Loss Factors using Point Mobilities," Philosophical transactions of the royal society A, 346, pp. 465-475.

18. Cuschieri J.M., and Sun J.C., 1994, "Use of Statistical Energy Analysis For Rotating Machinery, Part I: Determination of Dissipation and Coupling Loss Factors Using Energy Ratios," Journal of Sound and Vibration, 170, pp.181-190. 